\title{
Separation and Identification Main Polyphenolic Compounds From Urginea undulata
}

\author{
Abd El-Monem M. Sharaf ${ }^{1}$, Mohammed H. Elhaw ${ }^{1}$,Nashaat N. Kassem ${ }^{1}$ \\ ${ }^{\text {'}(B o t a n y}$ and Microbiology Department, Faculty of Science, Al-Azhar University)
}

\begin{abstract}
Chromatographic methods revealed the separation of eight biologically active constituents from Urgineaundulata. Bulb and Identification of the chemical composition as well as the physico-chemical properties of the eight active substances was carried out using Ultraviolet and ${ }^{1} H-N M R$ spectral data, the separated compounds are three phenolic acids (Ferulic acid, Gallic acid (3, 4, 5-trihydroxy benzoic acid) andCoutoric acid) and five compounds of flavonoids (4', 7-dihydroxyflavone, Quercetin, Rutin,Apigenin and Luteliolin-7, 3\, \, tri-O-glucuronide).
\end{abstract}

Keywords:Urgineaundulata, phenolics, bulbs, flavonoids, phenolic acids, U.V. and 1H NMR.

\section{Introduction}

Phytochemical constituents are the main source for the establishment of several pharmaceutical industries. The chemical constituents present in the plant play a significant role in the identification of crude drugs (Akindele\&Adeyemi, 2007). Probably originated in central Asia and then introduced in Europe by the Phoenicians around 2000 years ago, onion is grown in every part of the world because it is widely adaptable and can occupy a wide range of ecological niches. World onion production has increased by at least $25 \%$ over the past 10 years with current world production being about 85 million tons from 4.3 million hectares making it the second most important horticultural crop after tomatoes (Faostat, 2014). Onion is considered one of the most versatile vegetables: from raw to caramelized and from marinated to roasted, it can be found in a wide range of recipes from breakfast to supper and is accepted by almost all traditions and cultures. However, onion is not only a food, but it has also well-known medicinal and functional properties.The recent literature is rich in both in vivo and in vitro studies reporting the anti-thrombotic- (Lee et al., 2013), hypo-lipidemic(Lee et al., 2012; Srinivasan, 2013)anti-diabetic- (Jung et al., 2011), anti-obesity- (Yoshinariet al., 2012), antioxidant-(Alpsoyet al.,2012; Lee et al., 2012), antiinflammatory (Vazquez-Prietoet al., 2013), cancer chemopreventive- (Wang et al., 2012), and antiparasiticproperties of onion extracts (Klimpelet al., 2011; Aboelhadidet al.,2013). Moreover, some epidemiological studies suggest that a diet rich in onions may have a favorable effect on the risk of acute myocardial infarction (Galeone et al., 2009) and benign prostatic hyperplasia(Galeoneet al., 2007).

1. Plant Materials :

\section{Materials And Methods}

Fresh bulbs of Urgineaundulata were collected from growing habitats in Lakes Borollos (Elwardisland) rulbs ere dry and grinding to final powder for further investigation.

2. Methods:

* Preparation of Polyphenolic Compounds Extract

Phenolic compounds $750 \mathrm{gm}$ Dry powder of Urgineaundulata were extraction by $70 \%$ ethanol and purified according to standard procedures reported by Mabry et al., (1970) and Harborne, (1984). Combined filtrates were evaporated under reduced pressure using rotavapour apparatus until a minimum amount of solvent remained. The residue (greenish sticky) was stored in a refrigerator at $5{ }^{\circ} \mathrm{C}$ and kept for using in different analysis.

\subsection{Chromatographic Investigation}

\subsubsection{Paper Chromatography}

Two dimensional paper chromatography (TDPC) was carried out on Whatman (1MM) for comparative studies of aerial part extracts under investigation using BAW for the first dimension, followed by $15 \% \mathrm{AcOH}$ for the second dimension. For separation and purification of mixture of the phenolic compounds, the elution techniques on Whatman paper (3MM) were used. After the material under investigation was applied to the paper chromatography, the latter was run using solvent system No: 1-8 as outlined in table (1). Glass chromatographic tanks were used applying the ascending or descending techniques. The developed P.C. were air - dried and examined before spraying under both visible and UV light $(\lambda \max =366$ and $245 \mathrm{~nm})$. The P.C. were exposed to ammonia vapours for about 2-3 seconds and immediately re-examined the P.C. to observe any possible changes that may eventually appear in color or fluorescence under a long wave UV lamp. 


\subsubsection{ColumnChromatography}

The separation and identification of plant extracts were carried out by column chromatographic techniques (CC) (Markham, 1982). Silica gel columns were used for the separation and purification of phenolic compounds mixtures. Fractions produced from a large column often yielded simple mixtures of phenolic compounds. Further separation on small column or by paper and thin layer chromatography (T.L.C.) was carried out to obtain a pure flavonoids and phenolic acids.

\subsection{Identification Techniques of Flavonoids}

The qualitative analysis of flavonoid glycosides involves three distinct methods:

1- Identification of the aglycone.

2-Identification of the sugar moiety.

3- Identification of the point of the sugar attachment.

This is usually carried out through both chemical and physical investigation as indicated below.

\subsubsection{ChemicalAnalysis}

Analysis to determine the nature of aglycones and sugars was carried out according to standard procedures performed by Mabry et al., (1970) and Harborne (1984).

\section{*Complete Acid Hydrolysis}

Complete acid hydrolysis was carried out for 40-60 min. at $1000 \mathrm{C}$ using $5 \mathrm{ml} 2 \mathrm{~N}$-hydrochloric acid. The hydrolysis was then extracted with ethyl acetate, and the received extract was subjected to paper chromatographic investigation to detect the aglycones, the mother liquor was carefully neutralized, and then subject to chromatographic investigation by used HPLC to detect the sugars.

\section{- Identification of the Aglycone}

Identification of the aglycone moiety of flavonoid glycosides was based on direct co-chromatographic comparison with standard sample or/and UV spectroscopy followed NMR if needed.

\section{- Identification of the Sugar}

Sugars obtained by hydrolysis of the flavonoid glycosides were identified by chromatographic investigation using HPLC.

\subsubsection{PhysicalAnalysis}

Complete elucidation of the flavonoids should include physical methods such as ultraviolet (UV) and nuclear magnetic resonance (1H-NMR and 13C-NMR) measurements.

\subsection{2a. Ultraviolet Spectroscopic Analysis}

Ultraviolet visible absorption spectroscopy is perhaps the most useful technique available for flavonoid structure analysis using UV- visible spectrophotometer (Shimadzu model UV-240 and 2401 PC). The technique is used to aid both identification of the oxygenation and glycosylation patterns (Mabry et al., 1970 and Markham,1982). The flavonoid spectrum is usually determined in methanol using $2 \mathrm{ml}$ quartz cells. The spectrum typically consists of two absorption maxima at the ranges $240-285 \mathrm{~nm}$ (band II) and 300-500 nm (band I). The precise position and relative intensities of these maxima give valuable information on the nature of the flavonoid and its oxygenation pattern; the effects brought about by these changes are as follows:

1- Changes in the substitution of the A-ring tend to be reflected in the band II absorption while alternations in the substitution of the B-ring and C-ring tend to be more apparent from the band I absorption.

2- Additional oxygenation (especially hydroxylation) generally causes a shift of the appropriate band to longer wavelength, e.g. band I in 3,5,7,-tri $\mathrm{OH}$ flavone, $359 \mathrm{~nm}$; 3,5,7,4\-tetra $\mathrm{OH}$ flavone, $367 \mathrm{~nm} ; 3,5,7,3 \backslash, 4 \backslash$,-penta $\mathrm{OH}$ flavone, $370 \mathrm{~nm}$ and 3,5,7,3\,4\,5\-hexa $\mathrm{OH}$ flavone, $374 \mathrm{~nm}$.

3- Methylation or glycosylation (especially of 3, 5, 7, and 4\ hydroxyls) causes band shifts to shorter wavelength. The nature of the sugar in glycosides is normally of no consequence.

4- Acetylation tends to nullify the effect of a phenolic hydroxyl group on the spectrum.

5- The presence of cinnamic acid as acyl function on a flavonoid can be detected by the presence of an absorption band at $320 \mathrm{~nm}$ in flavonoids that themselves lack significant absorption at this region (e.g. anthocyanins).

6- In flavones and flavonols, the presence of 3\, 4\-di-OH system is generally evidenced by a second peak (sometimes a shoulder) in band II.

\subsection{2b. 1H-NMR spectroscopy}

The NMR spectroscopy is a well - established method for structure elucidation of flavonoids (Mabry et al., 1970) using a Jeol Ex-500 spectroscopy; 500MHz (1NMR), $125 \mathrm{MHz}$ (13C-NMR) or Joel JNM-EX 270 spectroscopy; $270 \mathrm{MHz}$ (1H-NMR), $67.5 \mathrm{MHz}$ (13C-NMR).

There are widely applied techniques for obtaining 1H-NMR spectra, using DMSO-d6 (hexadeutro dimethyl sulphoxide) as a solvent for the direct $1 \mathrm{H}-\mathrm{NMR}$ analysis.

1H-NMR spectra yield three sets of information: the integrals, the coupling pattern and the chemical shifts.

1 - The integrals define the number of protons represented by each signal or group of signals. 
2- The coupling patterns reflect the mutual arrangement of the coupling protons. In aromatic compounds, the coupling constants are 7-9 Hz between ortho protons, $1-3 \mathrm{~Hz}$ between meta protons and less than $1 \mathrm{~Hz}$ between para protons. The Para coupling, however, is usually not resolved, but causes broadening of the single only, which may in turn obscure a meta coupling.

3- The chemical shift of a proton indicates its chemical environment i.e. its position relative to any other part of the molecule. Chemical shift values are nowadays almost exclusively given parts per million (ppm) downfield from the TMS signal (Harborne, 1993). I.e. 1H-NMR analysis has been used to determine the aglycone part of the isolated flavone glycosides, to decide the number of sugars in the flavone O-glycosides, and the positions of the linkages of the sugar units in the falvone $\mathrm{C}$-glycosides.

The great number of published 1H-NMR spectra of flavonoids simplified the chemical shift assignments of the protons in the aromatic region of the flavonoid (Table 2). It also helps to decide the number of sugar units in the flavonoid O-glycosides, since the chemical shifts of the anomeric protons are more downfield than the other sugar protons.

For structure elucidation of flavonoid C-glycoside, measurements have been made on the parent flavonoid Cglycoside in DMSO-d6 (Mabry et al., 1970), and the same solvent could be used for both 1H and 13C-NMR spectroscopy. When only small amounts of a sample were available, the C-glycoside was dissolved in DMSOd6 and submitted to $1 \mathrm{H}-\mathrm{NMR}$ analysis followed by permethylation according to the mass spectroscopy analysis. By holding a high temperature $(800 \mathrm{C}$ ) when recording the 1H-NMR spectrum, the signal obtained from the absorbed water was minimal.

\subsection{2c. 13C-NMR Spectroscopy}

13C-NMR provides a very sensitive method for determination of the structure of complex compounds. With the introduction of Fourier-transform methods, 13C-NMR spectroscopy has developed into a powerful tool for the structure elucidation of small amounts of natural products. Over the past thirty years, 13C-NMR spectroscopy has become well established in the structure analysis of flavonoids. Several articles have been published. Most of them have dealt with flavonoid aglycones(Chari et al., 1977; Markham et al., 1978 and Shen et al., 1993) and only a few have dealt with C-glycosides (Chopin et al., 1978 and Agrawal, 1989). The position of a signal relative to the TMS reference $(0.0 \mathrm{ppm})$ is a good guide to the type of carbon represented. This position is shifted markedly by nearby substituents. Such effects are predictable in their extent and have led to the formulation of substituent effect data, which defines the expected effect (on aromatic carbon resonance) of introducing a new substituent into an aromatic ring. Using this type of substituent effect data, it is possible to calculate with great accuracy the spectrum of an unknown flavonoid form. To do this it is necessary to have available a wide range of reference spectra and a number of such complications have appeared.

In general terms sugar-related substitution include shifts are as follows:

1- O-glycosylation of a flavonoid hydroxyl produces an upfield shift of up to $2 \mathrm{ppm}$ in the signal of the adjacent flavonoid carbon and downfield shifts in the signal of the ortho and especially para related carbons (1-4 ppm).

2- C-glycosylation of a flavonoid aglycone causes a $10 \mathrm{ppm}$ downfield shift of the signal of the glycosylated carbon, but leaves other signals relatively unaffected.

3- Glycosylation of sugar by glucose causes an 8 ppm downfield shift in the signal of the glycosylated carbon accompanied by a 1-3 ppm upfield shift in the signal of the adjacent carbons. Glycosylation by rhamnose causes smaller shifts (3-6 ppm downfield and 1-2 ppm upfield, respectively).

Table 1.Approximate chemical shifts of various flavonoids proton types

\begin{tabular}{|c|c|}
\hline Chemical shift (ppm) & Proton type \\
\hline $0-0.5$ & Trimethylsilyl ether group \\
\hline c.1.0 & Rhamnose C-CH (broad doublet) \\
\hline c.1.7 & Prenyl (-CH $2-\mathrm{CH}=\mathrm{C}\left(\mathrm{CH}_{3}\right)_{2}$ methyl groups \\
\hline c.2.0 & Acetate $\left(-\mathrm{OCOCH}_{2}\right)$ and aromatic $\mathrm{C}-\mathrm{CH}_{2}$ \\
\hline $2-3$ & H-3 of flavanones (two proton - multiplet) \\
\hline $3.5-4.0$ & Most sugar protons \\
\hline $4.2-6.0$ & $\begin{array}{l}\text { H-1 of sugars (also H-2 of dihydroflavanolos, } 5.0 \text { ppm \& H-2 of flavanones 5-5.5 } \\
\text { ppm) }\end{array}$ \\
\hline c 6.0 & Methylenedioxy $\left(\mathrm{O}-\mathrm{CH}_{2}-\mathrm{O}\right)$ singlet \\
\hline $6.0-8.0$ & A and B- ring protons \\
\hline $7.5-8.0$ & H-2 of isoflavones (singlet) \\
\hline $12-14$ & 5-OH (observed only when using DMSO- $\mathrm{d}_{6}$ \\
\hline
\end{tabular}




\section{Phenolic Compounds}

\section{Results And Discussion}

Dried powder of Urgineaundulata total aerial parts $(500 \mathrm{~g}$.) in studied habitats, when extracted by ethanol $70 \%$ in soxhlet apparatus concentrated to very small volume by used rotavapour under reduce pressure at $40 \mathrm{Co}$ to yield $58 \mathrm{gm}$., fraction of Urgineaundulata under investigation was examined by using $\mathrm{AcOH}-15 \%$ and BAW, dried chromatograms examined under UV light, re-examined after exposure to ammonia then spread $\mathrm{AlCl} 3$ to complete evaluated phenolic compounds and chosen the best method for isolation and purification.

\subsection{Isolation and Identification of Polyphenolic Compounds of Urgineaundulata}

$70 \%$ total ethanol extract after examination by using $\mathrm{AcOH}-15 \%$ and $\mathrm{BAW}$, was applied on the top of

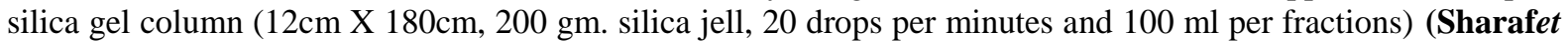
al., 2014) which started from hexane and increased in polarity., in system (hexan : petroleum ether 5:5 revealed the presence of some concentration compounds (fraction 11), which preparative in acetic acid $15 \%$ in whattmann paper chromatography $3 \mathrm{~mm}$ to yield one main blue band, which were subjected to spotting by paper chromatography to using solvent AcOH-15\% and BAW revealed the presence of one spot of phenolic acid in nature, compound N1 $58 \mathrm{mg}$. by increased in polarity in system hexan : petroleum ether 1:9 (fraction 17), which preparative in system chloroform :methanol 9:1 in thin layer chromatography TLC to yield one sharp blue band, which were subjected to spotting by paper chromatography to using solvent AcOH-15\% and BAW revealed the presence of one spot of phenolic acid in nature, compound $\mathrm{N} 235 \mathrm{mg}$. in system petroleum ether : chloroform 3:7 (fraction 26), which preparative in in thin layer chromatography TLC ( hexan : chloroform :methanol 2:4:4) to yield one sharp yellow band, which were subjected to spotting by paper chromatography to using solvent AcOH-15\% and BAW revealed the presence of one spot of flavonoids in nature, compound N3 $108 \mathrm{mg}$. while in system chloroform : ethyl acetate 8:2 (fraction 43) which was applied on the top of silica gel column $(5 \mathrm{~cm} \mathrm{X}$ $80 \mathrm{~cm}, 80 \mathrm{gm}$. silica jell, 20 drops per minutes and $50 \mathrm{ml}$ per fractions) which started from hexan and increased in polarity., revealed the presence of one main fractions, in system petroleum ether : chloroform 6:4, preparative of latter fraction in BAW (butanol :acetic acid : distal water 4:1:5) in whattmann paper chromatography $3 \mathrm{~mm}$ to yield one main yellow band, which were subjected to spotting by paper chromatography to using solvent $\mathrm{AcOH}-15 \%$ and $\mathrm{BAW}$ revealed the presence of one spot of flavonoids in nature, compound N4 $44 \mathrm{mg}$. in ethyl acetate : methanol 9:1 (fraction 52), after examination by using AcOH$15 \%$ and BAW was preparative application in paper chromatography 3MM (30 sheet) in BAW as a mobile phase, the presence yellow Band was applied on thin layer chromatography TLC in system TEF (toluene : ethyl acetate : formic acid 5:4:1) which yielded two main band, band 1 was sharp yellow band, elution by methanol to produced N5. $29 \mathrm{mg}$. while band 2 was phenolic acid in nature N6 57mg. at the end in system ethyl acetate : methanol $4: 6$ were subjected to spotting by paper chromatography to using solvent AcOH-15\% and BAW, latter fraction was applied on the top of silica gel column $(5 \mathrm{~cm} \mathrm{X} 80 \mathrm{~cm}, 85 \mathrm{gm}$. silica jell, 20 drops per minutes and $50 \mathrm{ml}$ per fractions) which started from ethyl acetate and increased in polarity., in system ethyl acetate : methanol 8:2, revealed the presence of one main fractions, which preparative of latter fraction in BAW (butanol :acetic acid : distal water 4:1:5) in whattmann paper chromatography $3 \mathrm{~mm}$ to yield two main band, band 1 was sharp yellow band, elution by methanol to produced N7. $48 \mathrm{mg}$. while band 2 was flavonoids in nature N8 59mg. which were subjected to spotting by paper chromatography to using solvent $\mathrm{AcOH}-15 \%$ and BAW to make purity test for specific compounds.

Table 2.Spectra data for main active constitutes isolated from of Urgineaundulata bulbs.

\begin{tabular}{|c|c|c|}
\hline NO. & SPECTRA DATA & STRUCTURE \\
\hline $\mathbf{1}$ & $\begin{array}{l}\text { Rf: Acetic acid 15\%: 0.87, BAW: 0.5, COLOR :U.V.: Blue, U.V. + } \\
\text { NH3: Blue, U.V.: MeOH: } 283 \text { sh, 318, NaOMe: } 284 \mathrm{sh}, 320, \mathrm{IH} \\
\text { NMR : (DMSO-D6), } \delta: 3.8(\mathrm{~s}, 3 \mathrm{H}, \mathrm{CH} 3), 4.9(\mathrm{~s}, 1 \mathrm{H}, \mathrm{Ar}-\mathrm{OH}), \\
\text { 6.45(d, } 1 \mathrm{H}, \mathrm{J}=7.8 \mathrm{~Hz}, \text { Alip-H), } 6.5(\mathrm{~m}, 2 \mathrm{H}, \mathrm{J}=4.8 \mathrm{~Hz}, \mathrm{Ar}-\mathrm{H}), 6.95(\mathrm{~d}, \\
1 \mathrm{H}, \mathrm{J}=2.7 \mathrm{~Hz}, \quad \text { Ar-H), } 7.5(\mathrm{~d}, 1 \mathrm{H}, \mathrm{J}=8.5 \mathrm{~Hz}, \quad \text { alip-H), } 11(\mathrm{~s}, 1 \mathrm{H} \text {, } \\
\text { COOH). , C10H10O4: Ferulic acid. }\end{array}$ & $\mathrm{HO}_{3}$ \\
\hline 2 & 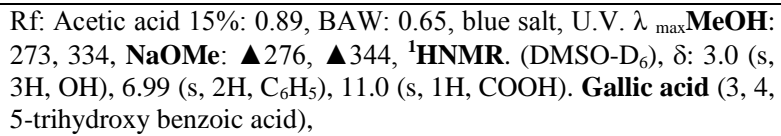 & \\
\hline
\end{tabular}




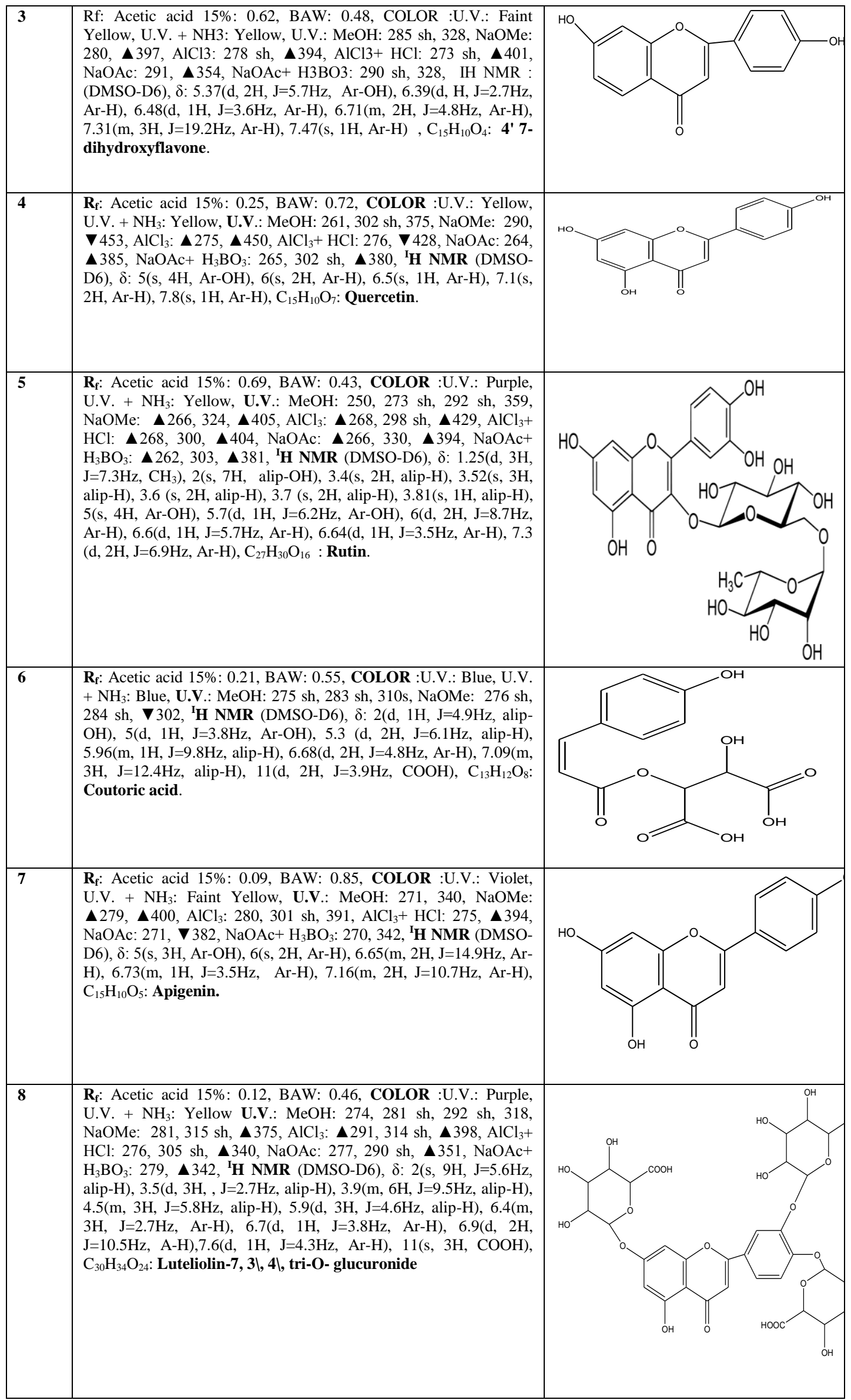


In the present studies, rutin was separated in pure form ethyl acetate and metanol fraction of Urgineaundulataand identification by use U.V. and ${ }^{1} \mathrm{H}$ - NMR, which clear that the rutin was content multi hydroxyl group attached with heterocyclic ring and four hydroxyl group attached to aliphatic sugar chain, this hydroxyl group refer to polar solvent behavior, "polar solvent dissolved polar solute and non-polar solvent dissolved non-polar solute" this is a rule in the solubility science, but result and rule according to observation was conflict, To explain this contrast is possible to say that the rutin attached with nonpolar compound e.g. lipid by bond and this bond was break by mobile phase with high elute strength according to that the rule may be modification by adding Condition that we should note conjugated compound attached by bonds (Sharaf et al., 2014).

\section{References}

[1] Aboelhadid, S., Kamel A., Arafa, W. and Shokier, K.(2013). 'Effect of Allium sativum and Allium cepa oils on different stagesofBoophilusannulatus', Parasitology Research, vol. 112, no. 5,pp.1883-1890.

[2] Agarwa, P., Sharma, B., Fatima, A. and Jain, S. (1989). An update on Ayurvedic herb Convolvulus pluricaulisChoisy. Asian Pac J. Trop Biomed; 4(3): 245-252.

[3] Akindele, A. and Adeyemi, O. (2007). Anti-inflammatory activities of the aqueous leaf extract of Byrsocarpuscoccineus. Fitoterapia, 78: 25-28

[4] Alpsoy, S., Aktas, C, Uygur, R., Topcu, B., Kanter, M., Erboga, M.,Karakaya, O.andGedikbasi, A. (2013). 'Antioxidant and anti- apoptotic effects of onion (Allium cepa) extract on doxorubicin-induced cardiotoxicity in rats', Journal of Applied Toxicology, vol. 33 , no. 3 , pp. 202-208.

[5] Chari, V., Wagner, H., and Neszmelyi, A., (1977). In "Farkas, L.; Gabor, M. and Kally, F. (Eds.). Flavonoids and Bioflavonoids".Elsevier, Ireland. PP. 293-301.

[6] Chopin, J., Buillant, M., Ramachandran Nair, A., Ramesh, P.and Mabry T. (1978). New C-glycosyl flavones from Mollugodistica. Phytochemistry, 17: 299-300.

[7] FAOST (2014). Food and Agriculture Organization of the united nation, viewed 27 August <http://faostat.fao.org/site/567/DesktopDefault. aspx?PageID=567>.

[8] Galeone, C., Pelucchi, C., Talamini, R., Negri, E., DalMaso, L.,Montella, M., Ramazzotti, V., Franceschi, S.andLa Vecchia, C. (2007). 'Onion and garlic intake and the odds of benign prostatic hyperplasia', Urology, vol. 70, no. 4, pp. 672-676.

[9] Galeone, C., Tavani, A., Pelucchi, C., Negri, E.and La Vecchia, C., (2009). 'Allium vegetable intake and risk of acute myocardial infarction inItaly', European Journal of Nutrition, vol. 48, no. 2, pp. 120-123.

[10] Harborne, J. (1984). In "Phytochemical Methods", Chapman and Hall. London, 287 pp.

[11] Harborne, J. (1993). In "The Flavonoids-Advanced in Research Science", Chapman and Hall. London, pp. 565-588.

[12] Jung, J., Lim, Y., Moon, M., Kim, J. and Kwon, O. (2011). 'Onion peel extracts ameliorate hyperglycemia and insulin resistance in high fat diet/streptozotocin-induced diabetic rats', Nutrition \& Metabolism, vol. 8, no. 18, pp.1-8.Chun, K.., Kim, D., and Lee, C.Y. (2013): Journal of Agriculture and Food chemistry, 51: 8067-8072.

[13] Klimpel, S., Abdel-Ghaffar, F., Al-Rasheid, K.,Aksu, G., Fischer, K.,Strassen, B.andMehlhorn, H. (2011).'The effects of different plant extracts on nematodes', Parasitology Research, vol. 108, no. 4, pp. 1047-1054

[14] Lee, S., Moon, J., Do, H., Chung, J., Lee, K., Cha, Y.and Shin, M. (2012). 'Onion peel extract increases hepatic low-density lipoprotein receptor and ATP-binding cassette transporter A1 messenger RNA expressions in Sprague-Dawley rats fed a highfat diet', NutritionResearch, vol. 32,no. 3, pp. 210-217.

[15] Lee, S., Moon, J., Chung, J., Cha, Y.and Shin, M. (2013).Effect of quercetin-rich onion peel extracts on arterial thrombosis in ratsFoodand Chemical Toxicology, vol. 57, pp. 99-105.

[16] Mabry, T., Markham, K., and Thomas, M. (1970). In "The Systematic Identification of Flavonoids." Springer-Verlag, New York, Heidlberg, Berlin, 354 pp.

[17] Markham, K.,Ternai, B., Stanley, R.., Geiger, H. and Mabry, T. (1978). Carbon-13NMR studies of flavonoids. Ш: Naturally occurring flavonoid glycosides and their acylated derivatives. Tetrahedron, 34 (9): 1389-1397.

[18] Markham, K., (1982). In "Techniques of Flavonoid Identification". Academic Press. New York, 113 pp.

[19] Shen, C., Chang, Y., and Ho, L. (1993). Nuclear magnetic resonance studies of 5, 7- dihydroxy flavonoids. Phytochemistry, 34(3): 843-845.

[20] Srinivasan, K. (2013).'Dietary spices as beneficial modulators of lipid profile in conditions of metabolic disorders and diseases', Food \&Function, vol. 4, no., pp. 503-521.

[21] Vazquez-Prieto, M., Rodriguez Lanzi, C.,Lembo, C., Galmarini, C.,andMiatello, R.(2013): 'Garlic and onion attenuates vascular inflammation and oxidative stress in fructose-fed rats', Journal of Nutrition and Metabolism, http://dx.doi.org/10.1155/2011/475216.

[22] Wang, Y., Tian, W.and Ma, X. (2012). 'Inhibitory Effects of onion(Allium cepa L.) extract on proliferation of cancer cells and adipocytes via inhibiting fatty acid synthase', Asian PacificOrganization for Cancer Prevention, vol. 13, no. 11, pp. 5573-5579.

[23] Yoshinari, O., Shiojima, Y.and Igarashi, K. (2012). 'Anti-Obesity Effectof Onion Extract in Zucker Diabetic Fatty Rats', Nutrients, vol. 4, no. 10, pp. 1518-1526. 